\title{
In vitro fragmentation performance of a novel, pulsed Thulium solid-state laser compared to a Thulium fibre laser and standard Ho:YAG laser
}

\author{
Lea Kraft ${ }^{1} \cdot$ Ralf Petzold $^{1} \cdot$ Rodrigo Suarez-lbarrola $^{1} \cdot$ Arkadiusz Miernik $^{1}$ (D)
}

Received: 22 April 2021 / Accepted: 8 December 2021 / Published online: 14 December 2021

(c) The Author(s) 2021

\begin{abstract}
The aim of this work was to compare the fragmentation efficiency of a novel, pulsed Thulium solid-state laser (p-Tm:YAG) to that of a chopped Thulium fibre laser (TFL) and a pulsed Holmium solid-state laser (Ho:YAG). During the fragmentation process, we used a silicone mould to fixate the hemispherical stone models under water in a jar filled with room-temperature water. Each laser device registered the total energy applied to the stone model to determine fragmentation efficiency. Our study examined laser settings with single pulse energies ranging from 0.6 to $6 \mathrm{~J}$ and pulse frequencies ranging from 5 to $15 \mathrm{~Hz}$. Similar laser settings were applied to explicitly compare the fragmentation efficiency of all three devices. We experimented with additional laser settings to see which of the three devices would perform best. The fragmentation performance of the three laser devices differed statistically significantly $(p<0.05)$. The average total energy required to fragment the stone model was 345.96 J for Ho:YAG, $372.43 \mathrm{~J}$ for p-Tm:YAG and $483.90 \mathrm{~J}$ for TFL. To fragment the stone models, both Ho:YAG and p-Tm:YAG needed similar total energy $(p=0.97)$. TFL's fragmentation efficiency is significantly lower than that of Ho:YAG and p-Tm:YAG. Furthermore, we found the novel p-Tm:YAG's fragmentation efficiency to closely resemble that of Ho:YAG. The fragmentation efficiency is thought to be influenced by the pulse duration. TFL's shortest possible pulse duration was considerably longer than that of Ho:YAG and p-Tm:YAG, resulting in Ho:YAG and p-Tm:YAG exhibiting better fragmenting efficiency.
\end{abstract}

Keywords Holmium laser $\cdot$ Laser lithotripsy $\cdot$ Pulsed Thulium laser $\cdot$ Thulium fibre laser $\cdot$ Urinary stone $\cdot$ Urolithiasis

$\begin{array}{ll}\text { Abbreviations } \\ \text { Ho:YAG } & \begin{array}{l}\text { Pulsed, holmium:yttrium aluminium garnet } \\ \text { solid-state laser }\end{array} \\ \text { p-Tm:YAG } & \begin{array}{l}\text { Pulsed thulium:yttrium aluminium garnet } \\ \text { solid-state laser } \\ \text { Chopped, thulium fibre laser }\end{array} \\ \text { TFL } & \text { Mean value } \\ \text { M } & \text { Standard deviation in Joule } \\ \text { SD }(\sigma) & \text { Single pulse energy in Joule } \\ \mathrm{E}_{\mathrm{SP}} & \text { Pulse repetition rate/pulse frequency in } \\ \mathrm{f} & \text { Hertz } \\ \mathrm{P} & \text { Single pulse power in Watt }\end{array}$

Arkadiusz Miernik

arkadiusz.miernik@uniklinik-freiburg.de

1 Department of Urology, Faculty of Medicine, University of Freiburg-Medical Centre, Hugstetter Str. 55, 79106 Freiburg, Germany

\section{Introduction}

Urinary stone disease is one of the most common diseases worldwide, affecting between 3 and $12 \%$ of the population. Its prevalence appears to be increasing in industrialised countries. In the USA, the incidence of stone disease has more than doubled since the late 1970s. The situation is similar in Japan, Germany and other European countries. A nationwide survey from 1979 to 2001 in Germany shows a threefold increase in its incidence and prevalence $(0.54-1.47 \%$ and $4.0-4.7 \%)$ [1]. Urolithiasis is thus considered a common condition. The USA's prevalence is about $12 \%$. The prevalence of wealth-related urinary stone disease is also rising in economically emerging countries. [2]

Over the last three decades, it has become the gold standard in laser lithotripsy to employ pulsed holmium solid-state laser (Ho:YAG) to treat renal, ureteral and bladder stones [3]. The most commonly used methods for intracorporeal stone disintegration are dusting and fragmenting. In general, the dusting technique refers to laser settings with low energy 
$(0.2-0.5 \mathrm{~J})$ and high frequency $(15-80 \mathrm{~Hz})$ that produce minute fragments that pass spontaneously [4-6]. The fragmentation technique, on the other hand, necessitates high-energy $(>1 \mathrm{~J})$ and low-frequency settings $(<10 \mathrm{~Hz})$ that create fragments requiring active retrieval $[4,7]$. There is a substantial body of research and clinical trials demonstrating the benefits of fragmentation over dusting, and vice versa. [4, 7-9]. This emphasises the importance of providing a laser capable of both dusting and stone fragmentation [10,11].

There has been a surge of interest in using the dusting technique for laser lithotripsy for several years. The availability of Ho:YAG with its higher frequency rates and the recently available Thulium fibre laser (TFL) with lower pulse energy settings (up to $25 \mathrm{~mJ}$ ) and higher frequencies (up to $1600 \mathrm{~Hz}$ ) have all contributed to this trend [12-16]. The novel, pulsed Tm:YAG solid-state laser (p-Tm:YAG) has the potential to turn the long-running controversy between competing lithotripsy techniques on its head. The performance of $\mathrm{p}-\mathrm{Tm}: \mathrm{YAG}$ in dusting has been investigated [17]. When fibres were moving at a rate of $1500 \mathrm{~mm} / \mathrm{min}$, p-Tm:YAG enabled a 12 to $17 \%$ improvement in dusting efficiency over Ho:YAG.

We compared the fragmentation performance of the p-Tm:YAG to the current gold standard Ho:YAG and the recently available TFL in this report. The fragmenting efficiency of the laser devices was compared using the same independent variables of pulse energy and pulse frequency for direct comparison. Additional laser settings, such as achieving the highest possible energy level, were chosen to assess the capability of each laser system in terms of fragmentation efficiency.

The laser emitted wavelength of each laser device, which affects the water absorption coefficient and, as a result, the optical penetration depth in water, is one parameter that may influence fragmentation efficiency. It will be left out of the debate because it is not investigated in this paper.

The peak power corresponding to the pulse duration is another feature that distinguishes these three types of laser devices used in laser lithotripsy. According to the manufacturer's specifications, TFL's pulse duration is up to seven times longer than that of p-Tm:YAG and 13 times longer than that of Ho:YAG, resulting in TFL's lower peak power. This disparity in pulse duration may have major effects on fragmentation efficiency. [16, 18]. According to current knowledge, higher laser peak powers may lead to faster stone fragmentation.

\section{Materials and methods}

The p-Tm:YAG and Ho:YAG used in the following experiments were the same as those used by Petzold et al. [17]. The TFL was used as the third laser device. Table 1 shows the specifications of all three laser devices.

For these experiments, we used a single-use laser fibre (Dornier SingleFlex® ${ }^{\circledR} 400 \mu \mathrm{m}$ of Dornier MedTech GmbH) with a diameter of $400 \mu \mathrm{m}$.

Stone model spheres made of gypsum and glass (KMP Kugelmanufaktur Pekruhl, Germering, Germany) were used to simulate the inhomogeneous structure of kidney stones. The spheres had a diameter of $12 \mathrm{~mm}$ and weighed approximately $85 \mathrm{~g}$ each. The spheres were sawed into hemispheres of similar size. The hemispheres were submerged in room temperature water for at least $4 \mathrm{~h}$ before the experiments began.

A jar filled with room temperature water and a silicone mould to attach the hemispherical stone models formed our experimental setup (Fig. 1). The stone model was

Table 1 Comparison of the laser devices, utilised in the following experiments, in terms of general and technical specification

\begin{tabular}{|c|c|c|c|}
\hline Parameter & Holmium solid-state laser & Thulium solid-state laser & Thulium fibre laser \\
\hline Operating mode & Pulsed & Pulsed & $\begin{array}{l}\text { Pulse generation } \\
\text { by chopping CW } \\
\text { laser beam }\end{array}$ \\
\hline Abbreviation & Ho:YAG & p-Tm:YAG & TFL \\
\hline Model & MEDILAS $®$ H SOLVO $® 35$ - old & Evaluation model & YLR-2000-U \\
\hline Manufacturer & $\begin{array}{l}\text { Dornier MedTech Laser GmbH, Wessling, } \\
\text { Germany }\end{array}$ & $\begin{array}{l}\text { Dornier MedTech Laser GmbH, } \\
\text { Wessling, Germany }\end{array}$ & $\begin{array}{l}\text { IPG Photonics @ } \\
\text { IRE-Polus, } \\
\text { Fryazino, Russia }\end{array}$ \\
\hline Wavelength & $2080 \mathrm{~nm}$ & $2013 \mathrm{~nm}$ & $1940 \mathrm{~nm}$ \\
\hline $\begin{array}{l}\text { Water absorption coefficient at } \\
1013 \text { bar and } 37^{\circ} \mathrm{C}\end{array}$ & $3 \mathrm{~mm}^{-1}[14]$ & $6.8 \mathrm{~mm}^{-1}[19]$ & $14 \mathrm{~mm}^{-1}[14]$ \\
\hline Pulse energies & $0.1-3.5 \mathrm{~J}$ & $0.1-3 \mathrm{~J}$ & $0.025-6 \mathrm{~J}$ \\
\hline Pulse durations & $0.14-0.45 \mathrm{~ms}$ & $0.15-1.2 \mathrm{~ms}$ & $0.05-12 \mathrm{~ms}$ \\
\hline Pulse frequencies & $3-25 \mathrm{~Hz}$ & $5-200 \mathrm{~Hz}$ & $6-1600 \mathrm{~Hz}$ \\
\hline Maximum average power & $35 \mathrm{~W}$ & $120 \mathrm{~W}$ & $40 \mathrm{~W}$ \\
\hline
\end{tabular}


Fig. 1 (a) Jar filled with room-temperature water; (b) silicone moulds to attach the hemispherical stone model; (c) hemispherical stone model attached to silicone mould; (d) originally attached hemispherical stone model fragmented into four pieces; (e) hand-guided movement of the laser fibre indicated by the dotted lines
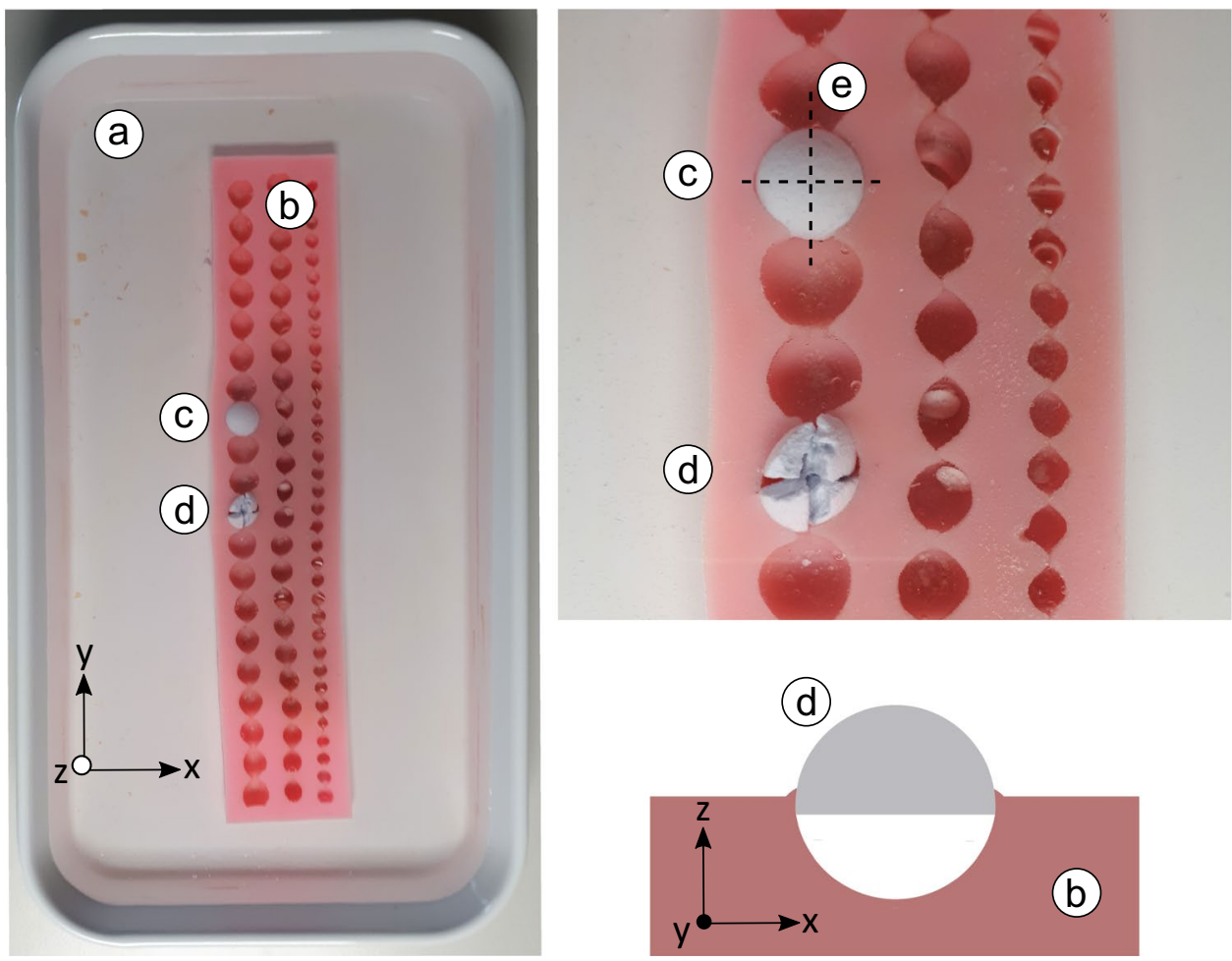

fragmented into four parts by moving the laser fibre in a cruciform path by hand. The hand-guided movement of the laser fibre was purposefully chosen in order to keep it in contact with the stone model at all times and to replicate the clinical approach to fragmenting a urinary stone. The laser fibre was adjusted in the depth of the groove created through cruciformly ablating the hemispherical stone models. An electronically operated feed, such as with an XY plotter, on the other hand, cannot ensure contact between the laser fibre tip and the stone model, which might reduce total efficacy.

During the fragmentation process, each laser system recorded the total energy required before the hemispheric stone model was fragmented into four pieces of roughly equal size. The fragmentation process was repeated five times with the same laser device at each laser setting. The mean total energy was calculated for each laser setting, yielding the mean total energies $\mathrm{E}_{1}$ (Ho:YAG), $\mathrm{E}_{2}$ (p-Tm:YAG) and $\mathrm{E}_{3}$ (TFL).

For the fragmentation technique, we chose standard laser settings $\left(\mathrm{E}_{\mathrm{SP}}=0.6-3 \mathrm{~J}, f=5-15 \mathrm{~Hz}\right)$ and the shortest possible pulse duration. All three laser devices alternated in performing five experimental runs per laser setting. The laser settings we selected are shown in Table 2.

Additionally, a few laser settings were chosen to measure each laser device's capability individually (Table 3). The pulse durations varied widely between the laser devices.
Table 2 The table illustrates laser settings used by all three laser devices specified by the single pulse energy $\left(\mathrm{E}_{\mathrm{SP}}\right)$, the pulse frequency (f) and resulting pulse power $\left(P=E_{S P} \bullet f\right)$. Each laser device's pulse duration $\tau$ is indicated

\begin{tabular}{llllll}
\hline $\mathrm{E}_{\mathrm{SP}}[\mathrm{J}]$ & $\mathrm{f}[\mathrm{Hz}]$ & $\mathrm{P}[\mathrm{W}]$ & $\begin{array}{l}\tau_{1} \\
(\mathrm{Ho}: Y A G) \\
{[\mathrm{ms}]}\end{array}$ & $\begin{array}{l}\tau_{2} \\
(\mathrm{p}-\mathrm{Tm}: \mathrm{YAG}) \\
{[\mathrm{ms}]}\end{array}$ & $\tau_{3}(\mathrm{TFL})[\mathrm{ms}]$ \\
\hline 0.6 & 10 & 6 & 0.22 & 0.174 & 1.2 \\
1 & 10 & 10 & 0.27 & 0.276 & 1.8 \\
2 & 5 & 10 & 0.37 & 0.648 & 3.9 \\
2 & 10 & 20 & 0.36 & 0.534 & 3.9 \\
3 & 5 & 15 & 0.45 & 0.81 & 6 \\
3 & 10 & 30 & 0.44 & 0.792 & 6 \\
\hline
\end{tabular}

The laser fibre was cleaved before each repetition, and the energy of a single pulse $\mathrm{E}_{\mathrm{SP}}$ was measured using a laser energy meter (StarBright ${ }^{\circledR}$, Ophir Spiricon Europe GmbH, Darmstadt, Germany). The distance between the laser fibre's tip and the radiation-sensitive region of the pyroelectric energy meter (Ophir ${ }^{\circledR}$ FPE80BF-DIF-C) was $55 \mathrm{~mm}$. A variance of less than $50 \mathrm{~mJ}$ was deemed permissible. Otherwise, the fibre was re-cut or a new one was used.

The statistical assessment involved calculating the mean value and standard deviation, as well as presenting the data graphically. Python (Python Software Foundation License) was used to analyse our results. The Shapiro-Wilk test 
Table 3 The table below shows laser settings, specified by the single pulse energy $\left(E_{S P}\right)$, pulse frequency $(f)$, resulting pulse power $\left(P_{S P}=\right.$ $\left.\mathrm{E}_{\mathrm{SP}} \bullet \mathrm{f}\right)$ and pulse duration $(\tau)$ of different laser devices

\begin{tabular}{lllll}
\hline Laser device & $\mathrm{E}_{\mathrm{SP}}[\mathrm{J}]$ & $\mathrm{f}[\mathrm{Hz}]$ & $\mathrm{P}[\mathrm{W}]$ & $\tau[\mathrm{ms}]$ \\
\hline Ho:YAG & 1 & 5 & 5 & 0.27 \\
& 3.5 & 10 & 35 & 0.45 \\
p-Tm:YAG & 1 & 5 & 5 & 0.334 \\
& 3 & 15 & 45 & 0.81 \\
TFL & 1 & 6 & 6 & 1.8 \\
& 3.5 & 10 & 35 & 7 \\
& 6 & 5 & 30 & 12 \\
& 6 & 6.7 & 40.2 & 12 \\
\hline
\end{tabular}

was used to determine data distribution. Levene's test was used to establish homogeneity of variances. Kruskal-Wallis $H$-test was used to determine the impact of the laser device on total energy $(E)$. Dunn's test was used as post hoc analysis to seek any indications of a substantial difference in fragmentation efficiency between Ho:YAG, p-Tm:YAG and TFL. Each laser device's impact on total energy $(E)$ was determined using either one-way analysis of variance (ANOVA) or Kruskal-Wallis $H$-test, depending on Levene's test and Shapiro-Wilk test. Tukey's HSD test or Dunn's test (based on the Bonferroni correction principle) was used for post hoc analysis. All analyses had a significance level of 0.05 .

\section{Results}

The pulse durations of TFL are clearly longer for each laser setting compared to Ho:YAG and/or p-Tm:YAG, as the diagrams show (Fig. 2).

Figure 2a shows each laser device's average total energy $(E)$ required to fragment a hemispherical stone phantom into four pieces, based on the laser settings from Table 2 and pulse duration $(\tau)$. The mean total energy of Ho:YAG, p-Tm:YAG and TFL was $346 \mathrm{~J}$ (standard deviation: $\mathrm{SD}=70 \mathrm{~J}), 372 \mathrm{~J}(\mathrm{SD}=92 \mathrm{~J})$ and $484 \mathrm{~J}(\mathrm{SD}=125 \mathrm{~J})$, respectively. Total energies recorded by $\mathrm{p}-\mathrm{Tm}$ :YAG were normally distributed $(W=0.93, p=0.05)$, in comparison to that of Ho:YAG $(W=0.92, p=0.04)$ and TFL $(W=0.89, p=0.004)$.
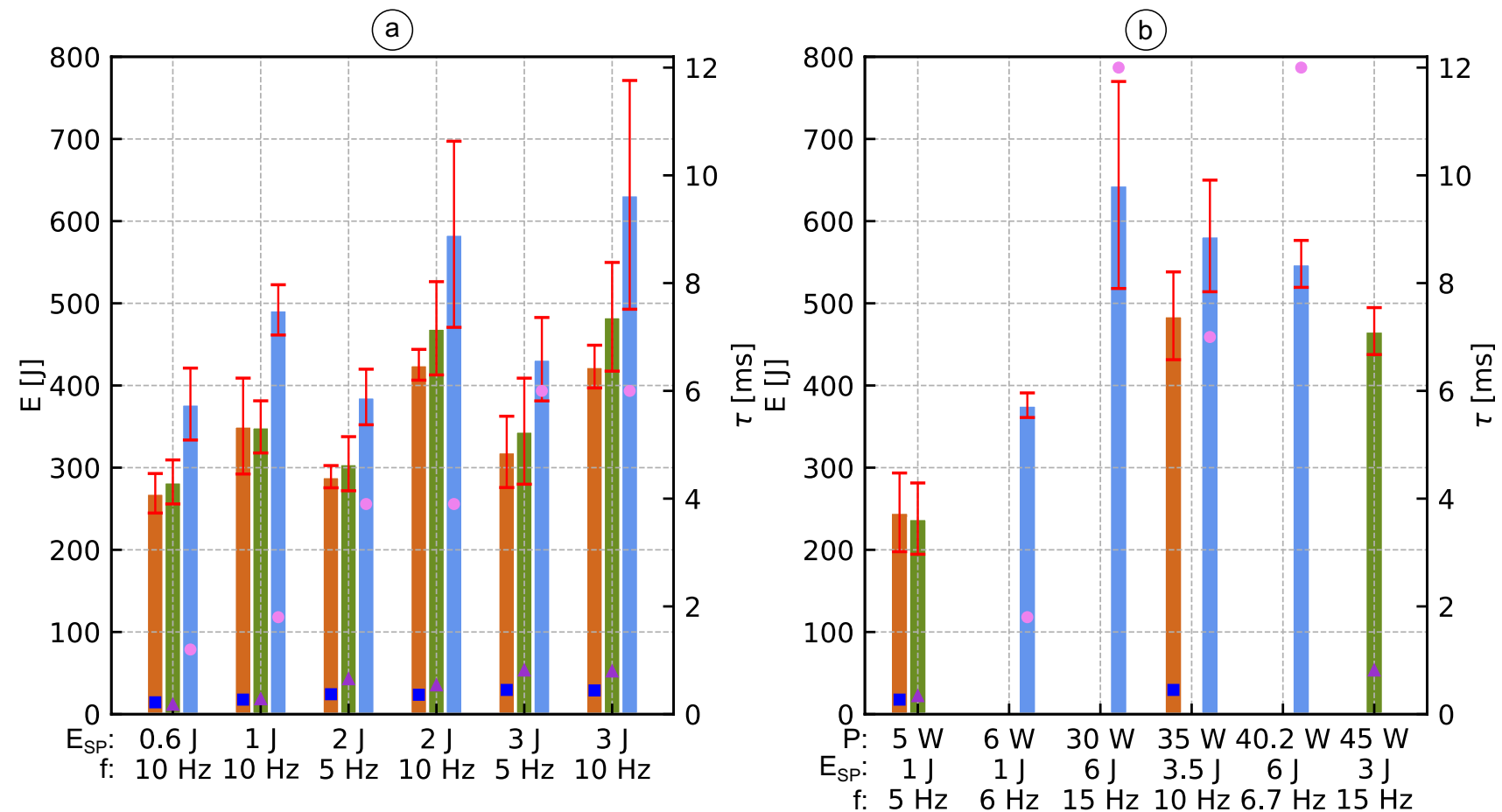

Legend:

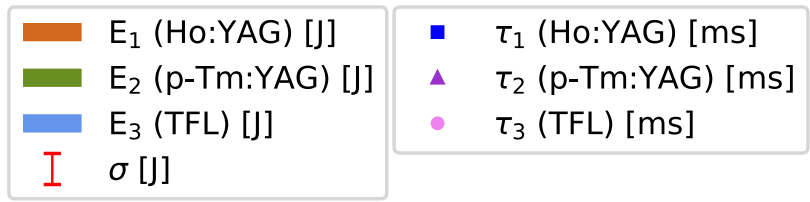

f: $5 \mathrm{~Hz} \quad 6 \mathrm{~Hz} \quad 15 \mathrm{~Hz} \quad 10 \mathrm{~Hz} \quad 6.7 \mathrm{~Hz} 15 \mathrm{~Hz}$

Fig. 2 The left $y$-axis shows the mean total energy $\left(E_{1}, E_{2}, E_{3}\right)$ of each laser device, based on the laser settings from Table 2 (a) as well as Table $3(\mathbf{b})$. The standard deviation $(\sigma)$ of five experimental runs of each laser device for each laser settings is given as red line. The pulse durations $\left(\tau_{1}, \tau_{2}, \tau_{3}\right)$ of the different laser settings in milliseconds, depending on the laser device, is placed at the right $y$-axis 
Equal variances were assumed $(F=1.40, p=0.25)$. According to Kruskal-Wallis $H$-test, there is a significant difference between the laser devices' main tendencies $(H=22.98$, $p=1 \mathrm{e}-05)$. TFL varies substantially from both Ho:YAG $(p=1 \mathrm{e}-05)$ and $\mathrm{p}-\mathrm{Tm}$ :YAG $(p=0.001)$, according to post hoc Dunn's test. There was no substantial difference between p-Tm:YAG and Ho:YAG $(p=0.97)$.

In conclusion, at all laser settings, Ho:YAG and p-Tm:YAG need less mean total energy than TFL. To fragment the artificial stones, both Ho:YAG and p-Tm:YAG require a similar mean total energy $(p=0.97)$.

Another of our findings was that increased pulse energy and/or pulse frequency does not always imply better stone fragmentation.

In addition, we investigated whether there was any statistically significant difference between each laser device's total energy and laser setting. Table 4 shows each laser setting's mean total energy of Ho:YAG $\left(E_{1}\right)$, p-Tm:YAG $\left(E_{2}\right)$ and TFL $\left(\mathrm{E}_{3}\right)$, as well as the standard deviation (SD). The total energies of each laser device's setting were distributed normally. Equal variances were assumed for Ho:YAG $(F=1.27, p=0.31)$, p-Tm:YAG $(F=0.52, p=0.76)$ and TFL $(F=2.39, p=0.07)$. According to one-way ANOVA, the total energy and laser settings of Ho:YAG $(F=15.02$, $p=1 \mathrm{e}-06), \mathrm{p}-\mathrm{Tm}: \mathrm{YAG}(F=11.78, p=8 \mathrm{e}-06)$ and TFL $(F=6.89, p=4 \mathrm{e}-04)$ were statistically different.

Post hoc Tukey's HSD test revealed differences in each laser device's various laser settings: For Ho:YAG, we observed significant differences between the mean total energies of laser settings $0.6 \mathrm{~J} / 10 \mathrm{~Hz}$ and $1 \mathrm{~J} / 10 \mathrm{~Hz}(p=0.027)$, $0.6 \mathrm{~J} / 10 \mathrm{~Hz}$ and $2 \mathrm{~J} / 10 \mathrm{~Hz}(p=0.001), 0.6 \mathrm{~J} / 10 \mathrm{~Hz}$ and $3 \mathrm{~J} / 10 \mathrm{~Hz}(p=0.001), 2 \mathrm{~J} / 5 \mathrm{~Hz}$ and $2 \mathrm{~J} / 10 \mathrm{~Hz}(p=0.001)$, $2 \mathrm{~J} / 5 \mathrm{~Hz}$ and $3 \mathrm{~J} / 10 \mathrm{~Hz}(p=0.001), 2 \mathrm{~J} / 10 \mathrm{~Hz}$ and $3 \mathrm{~J} / 5 \mathrm{~Hz}$ $(p=0.003), 3 \mathrm{~J} / 5 \mathrm{~Hz}$ and $3 \mathrm{~J} / 10 \mathrm{~Hz}(p=0.003)$. For p-Tm:YAG, there were significant differences between the mean total energies of laser settings $0.6 \mathrm{~J} / 10 \mathrm{~Hz}$ and $2 \mathrm{~J} / 10 \mathrm{~Hz}(p=0.001), 0.6 \mathrm{~J} / 10 \mathrm{~Hz}$ and $3 \mathrm{~J} / 10 \mathrm{~Hz}(p=0.001)$, $1 \mathrm{~J} / 10 \mathrm{~Hz}$ and $2 \mathrm{~J} / 10 \mathrm{~Hz}(p=0.03), 1 \mathrm{~J} / 10 \mathrm{~Hz}$ and $3 \mathrm{~J} / 10 \mathrm{~Hz}$ $(p=0.009), 2 \mathrm{~J} / 5 \mathrm{~Hz}$ and $2 \mathrm{~J} / 10 \mathrm{~Hz}(p=0.001), 2 \mathrm{~J} / 5 \mathrm{~Hz}$ and $3 \mathrm{~J} / 10 \mathrm{~Hz}(p=0.001), 2 \mathrm{~J} / 10 \mathrm{~Hz}$ and $3 \mathrm{~J} / 5 \mathrm{~Hz}(p=0.02)$, $3 \mathrm{~J} / 5 \mathrm{~Hz}$ and $3 \mathrm{~J} / 10 \mathrm{~Hz}(p=0.006)$. For TFL, there were significant differences between the mean total energies of laser settings $0.6 \mathrm{~J} / 10 \mathrm{~Hz}$ and $2 \mathrm{~J} / 10 \mathrm{~Hz}(p=0.01), 0.6 \mathrm{~J} / 10 \mathrm{~Hz}$ and $3 \mathrm{~J} / 10 \mathrm{~Hz}(p=0.002), 2 \mathrm{~J} / 5 \mathrm{~Hz}$ and $2 \mathrm{~J} / 10 \mathrm{~Hz}(p=0.02)$, $2 \mathrm{~J} / 5 \mathrm{~Hz}$ and $3 \mathrm{~J} / 10 \mathrm{~Hz}(p=0.003), 3 \mathrm{~J} / 5 \mathrm{~Hz}$ and $3 \mathrm{~J} / 10 \mathrm{~Hz}$ $(p=0.02)$.

Figure $2 \mathrm{~b}$ depicts each laser device's average total energy $(E)$ required to fragment the hemispherical stone model, based on the laser settings in Table 3 and pulse duration $(\tau)$. These laser settings were chosen to test fragmentation efficiency at each laser device's technical limits.

Higher single-pulse energies do not inherently result in higher fragmentation efficiency when comparing the laser settings of $6 \mathrm{~J} / 15 \mathrm{~Hz} / 30 \mathrm{~W}, 6 \mathrm{~J} / 6.7 \mathrm{~Hz} / 40.2 \mathrm{~W}$ and $3 \mathrm{~J} / 15 \mathrm{~Hz} / 45 \mathrm{~W}$, as shown in Fig. 2b. In general, TFL needs more energy to fragment the stone model than Ho:YAG and p-Tm:YAG, based on the mean total energy.

Taking into account that TFL's pulse frequency at laser setting $1 \mathrm{~J} / 6 \mathrm{~Hz} / 6 \mathrm{~W}$ is $1 \mathrm{~Hz}$ higher than that of Ho:YAG and $\mathrm{p}-\mathrm{Tm}$ :YAG at the $1 \mathrm{~J} / 5 \mathrm{~Hz} / 5 \mathrm{~W}$ laser setting; note that the Ho:YAG and p-Tm:YAG exhibit similar fragmentation efficiency, and both need less mean total energy than TFL as in Fig. 2b.

\section{Discussion}

This study investigated the fragmentation performance of a p-Tm:YAG in comparison to a Ho:YAG and TFL. Stone fragmentation performance is known to be influenced by parameters such as the single pulse energy $\left(\mathrm{E}_{\mathrm{SP}}\right)$, pulse frequency $(f)$ and pulse duration $[7,16,18]$. We compared the laser devices applying similar single pulse energies and pulse frequencies. The pulse duration for each setting could not be chosen to be identical due to fundamental differences in laser technology and pulse generation. Whenever possible, we were careful to select the shortest possible pulse duration for each laser device at each laser setting to enable the highest possible peak power. Additional laser settings were chosen to examine the capabilities of each laser device (e.g. high pulse energies in TFL).

Our first important finding was that the Ho:YAG and p-Tm:YAG needed less mean total energy to fragment the stone model in the same way as TFL. We made this
Table 4 The mean total energy (E) and standard deviation (SD) in Joule for each laser device (Ho:YAG, p-Tm:YAG and TFL) as well as for each laser setting, specified by the single pulse energy $\left(E_{S P}\right)$ in Joule, the pulse frequency (f) in Hertz and the single pulse power $(\mathrm{P})$ in Watt

\begin{tabular}{|c|c|c|c|c|c|c|c|c|}
\hline \multirow[t]{2}{*}{$\mathrm{E}_{\mathrm{SP}}[\mathrm{J}]$} & \multirow[t]{2}{*}{$\mathrm{f}[\mathrm{Hz}]$} & \multirow[t]{2}{*}{$\mathrm{P}[\mathrm{W}]$} & \multicolumn{2}{|c|}{ Ho:YAG } & \multicolumn{2}{|c|}{ p-Tm:YAG } & \multicolumn{2}{|l|}{ TFL } \\
\hline & & & $\mathrm{E}_{1}[\mathrm{~J}]$ & $\mathrm{SD}[\mathrm{J}]$ & $\mathrm{E}_{2}$ & $\mathrm{SD}[\mathrm{J}]$ & $\mathrm{E}_{3}$ & $\mathrm{SD}[\mathrm{J}]$ \\
\hline 0.6 & 10 & 6 & 268.8 & 24.0 & 282.6 & 26.8 & 377.4 & 43.8 \\
\hline 1 & 10 & 10 & 350.6 & 58.4 & 349.6 & 31.7 & 492.0 & 30.6 \\
\hline 2 & 5 & 10 & 289.0 & 13.5 & 304.8 & 32.9 & 386.0 & 33.8 \\
\hline 2 & 10 & 20 & 425.2 & 18.7 & 469.6 & 56.7 & 584.0 & 113.2 \\
\hline 3 & 5 & 15 & 319.2 & 43.4 & 344.4 & 64.5 & 432.0 & 50.8 \\
\hline 3 & 10 & 30 & 423.0 & 26.0 & 483.6 & 66.1 & 632.0 & 139.2 \\
\hline
\end{tabular}


observation in conjunction with various laser settings using pulse energies ranging from 0.6 to $3 \mathrm{~J}$ and frequencies ranging from $5 \mathrm{~J}$ to $10 \mathrm{~Hz}$. From the clinical point of view, lowering the total laser energy applied to the urinary tract during an endoscopic stone removal procedure can lower the risk of thermal injuries. Advanced laser technologies, according to Williams et al. [20], allow for higher output power (energy per second), which increases the risk of thermal tissue damage caused by heating the irrigation fluid within the urinary tract. Hein et al. [21, 22] emphasise that there is thermal damage potential in combination with insufficient irrigation rates even at low-power (low-energy and/or lowfrequency) settings.

Furthermore, our research discovered that the p-Tm:YAG's fragmentation efficiency is statistically equal to that of Ho:YAG $(p=0.97)$ and outperforms the TFL's $(p=0.001)$. To the best of our knowledge, this is the first study in urologic research to compare the fragmentation efficiency of three different laser technologies: Ho:YAG, TFL and the novel p-Tm:YAG, which is currently awaiting approval.

Secondly, we observed that TFL has a longer pulse duration and therefore lower peak power than Ho:YAG and p-Tm:YAG at all laser settings. These are the most likely causes of the lower fragmentation efficiency we observed.

Wezel et al. [18] reported that, in two different types of Ho:YAG, reducing the pulse duration from 700 to $350 \mu \mathrm{s}$ results in more thorough stone disintegration, two different stone compositions and two different fibre diameters.

According to Bader et al. [23], the fragmentation efficiency of a Ho:YAG at equal power settings between short and long pulse durations requires similar amounts of time before the stone models are sufficiently disintegrated. Dust weight and laser activation time served as measurement variables. They point out that there is no consensus as to what stone-particle size constitutes genuine 'stone dust'. Dust weight is not a universal metric, since it depends on factors such as fragment size. Since we wanted to eliminate the influence of frequency, we consciously distanced our work from a time-dependent measurement variable such as laser activation time. As a result, we chose to focus solely on the effects of laser pulse energy.

Another potential drawback is employing just one type of laser device, with pulse durations varying at the same single pulse energy and pulse frequency. The long pulse had a pulse duration 1.5 to 2.6 times longer than the short pulse. For single pulse energies $\geq 1 \mathrm{~J}$, the pulse durations of Ho:YAG and p-Tm:YAG were 1.5 to 6 times longer than that of TFL in our experiments.
Bell et al. [24] compared the fragmentation times of two separate Ho:YAG laser devices, one with low power and the other with high power. Regardless of pulse duration, they discovered that the high-power laser device completed the stone fragmentation procedure in half the time that the lowpower laser device required. This indicates that factors other than pulse duration, such as the energy applied, may have affected fragmentation efficiency. Treatment time before fragmentation was established as one of the calculated variables. As a measurement variable, treatment time may thus be influenced more than the total energy measured by the laser device, for example, by the pedal activation time. Aldoukhi et al. [25] suggested that the relationship between laser activation time and lithotripsy time, including pedal non-activation periods, be taken into account.

Alghamdi et al. [26] recently compared three different Ho:YAG pulse shapes in terms of stone disintegration versus total operating time. The shape of the laser pulses varied at the beginning of the laser pulse. Their findings suggest that, in addition to pulse duration, pulse shape can influence fragmentation efficiency.

We also observed that to fragment a stone, more total energy is required at constant energy levels and higher frequencies. We believe that the laser fibre is harder to control at higher frequency settings: as they create more dust, visibility is reduced, thus raising the risk of tissue perforation [7].

Our experiments may have been affected by certain primarily unseen circumstances. Firstly, the pulse duration at similar laser settings varied in each laser device for technical reasons. Furthermore, dividing the spherical stone models into two hemispheres may have resulted in hemispheres of slightly different size. Finally, to obtain replicable outcomes, we used artificial stone models. The composition, crystalline structure and scale of natural human stone samples vary significantly [27].

Further experimental studies and observations of the various principles of action of these laser systems are needed to enable clinically more robust recommendations.

\section{Conclusion}

According to our observations, the TFL's fragmentation efficiency was significantly lower than that of Ho:YAG and p-Tm:YAG. Furthermore, the fragmentation efficiency of p-Tm:YAG proved to be very similar to that of Ho:YAG. We found the TFL's shortest possible pulse duration to be 
considerably longer than that of Ho:YAG and p-Tm:YAG. That factor alone may have been the primary cause of the TFL's poor fragmentation performance. Clinical trials are needed to confirm these experimental findings.

Author contribution Conceptualization: RP, AM; material preparation, data collection and analysis, writing - original draft preparation: LK; writing-review and editing: RP, RSI, AM; funding acquisition, supervision: AM.

Funding Open Access funding enabled and organized by Projekt DEAL. The study was conducted as a collaborative research project with the medical technology company Dornier MedTech Laser GmbH, Wessling, Germany.

Data availability The raw data is with the corresponding author and can be provided on request.

Code availability The computer software for statistical analysis is with the corresponding author and can be provided on request.

\section{Declarations}

Ethics approval The manuscript contains no clinical studies or patient data, nor does it contain any studies with human or animal subjects performed by the any of the authors.

Conflict of interest A. Miernik receives research funding from the German Federal Ministry of Education and Research, Berlin (D). He receives support for his travel activities from the European Society of Urology, Arnhem (NL) and German Society of Urology, Düsseldorf (D). A. Miernik is also a consultant for: KLS Martin, Tuttlingen (D), Avateramedical, Jena (D), LISA LaserProducts GmbH, KatlenburgLindau (D), Schoelly fiberoptics GmbH, Denzlingen (D), Dornier MedTech Laser GmbH (D), Medi-Tate Ltd. (IL, USA) and b.braun Newventures GmbH, Freiburg (D). A. Miernik is speaker for the companies Richard Wolf GmbH (D) and Boston Scientific (USA). He also served as a reviewer for the Ludwig Boltzmann Gesellschaft, Wien (A). A. Miernik is involved in numerous patents and inventions in the field of medical technology. R. Petzold and L. Kraft have provided consulting services for Dornier MedTech Laser GmbH, Weßling (D). R. Suarez has no conflicts of interest to declare.

Open Access This article is licensed under a Creative Commons Attribution 4.0 International License, which permits use, sharing, adaptation, distribution and reproduction in any medium or format, as long as you give appropriate credit to the original author(s) and the source, provide a link to the Creative Commons licence, and indicate if changes were made. The images or other third party material in this article are included in the article's Creative Commons licence, unless indicated otherwise in a credit line to the material. If material is not included in the article's Creative Commons licence and your intended use is not permitted by statutory regulation or exceeds the permitted use, you will need to obtain permission directly from the copyright holder. To view a copy of this licence, visit http://creativecommons.org/licenses/by/4.0/.

\section{References}

1. Hesse A et al (2003) Study on the prevalence and incidence of urolithiasis in Germany comparing the years 1979 vs. 2000. Eur Urol 44:709-13

2. Trinchieri A (2008) Epidemiology of urolithiasis: an update. Clin Cases Miner Bone Metab 5:101-106

3. Bell JR, Penniston KL, Nakada SY (2017) In vitro comparison of stone fragmentation when using various settings with modern variable pulse holmium lasers. J Endourol 31(10):1067-1072

4. El-Nahas AR et al (2019) Dusting versus fragmentation for renal stones during flexible ureteroscopy. Arab J Urol 17(2):138-142

5. Tracey J et al (2018) Ureteroscopic high-frequency dusting utilizing a 120-W holmium laser. J Endourol 32(4):290-295

6. Doizi S, Keller EX, De Coninck V, Traxer O (2018) Dusting technique for lithotripsy: what does it mean?. Nat Rev Urol 15(11):653-654

7. Black KM, Aldoukhi AH, Ghani KR (2019) A users guide to holmium laser lithotripsy settings in the modern era. Front Surg $6: 48$

8. Matlaga BR et al (2018) Ureteroscopic laser lithotripsy: a review of dusting vs fragmentation with extraction. J Endourol 32(1):1-6

9. Hardy LA et al (2018) Fragmentation and dusting of large kidney stones using compact, air-cooled, high peak power, 1940-nm, Thulium fiber laser. Therapeutics and diagnostics in urology 2018. International Society for Optics and Photonics

10. Santiago JE et al (2017) To dust or not to dust: a systematic review of ureteroscopic laser lithotripsy techniques. Curr Urol Rep 18(4):32

11. Wenzel M, Bultitude M, Salem J (2019) Dusting, fragmenting, popcorning or dustmenting? Curr Opin Urol 29(2):108-112

12. Schembri M et al (2020) Thulium fiber laser: the new kid on the block. Turk J Urol 46(Supp. 1):S1-s10

13. Becker A et al (2020) Der "Super Pulse" Thulium Faser Laser Neuerungen im Bereich der Endourologie. UroForum 09:5

14. Traxer O, Keller EX (2019) Thulium fiber laser: the new player for kidney stone treatment? A comparison with Holmium:YAG laser. World J Urol 38(8):1883-1894

15. Hardy LA, Vinnichenko V, Fried NM (2019) High power holmium:YAG versus thulium fiber laser treatment of kidney stones in dusting mode: ablation rate and fragment size studies. Lasers Surg Med 51(6):522-530

16. Enikeev D et al (2020) A review of thulium-fiber laser in stone lithotripsy and soft tissue surgery. Curr Opin Urol 30(6):853-860

17. Petzold R, Miernik A, Suarez-Ibarrola R (2020) In vitro dusting performance of a new solid state thulium laser compared to holmium laser lithotripsy. J Endourol

18. Wezel F et al (2010) Effect of pulse energy, frequency and length on holmium: yttrium-aluminum-garnet laser fragmentation efficiency in non-floating artificial urinary calculi. J Endourol 24(7): 1135-1140

19. Vinnichenko V et al (2018) Comparison of a novel 450-nm laser with Ho:YAG (2100 nm), Tm fiber (1940 nm), and KTP (532 nm) lasers for soft-tissue ablation. SPIE BiOS. 10468: SPIE

20. Williams JG et al (2020) A temperature model for laser lithotripsy. World J Urol 39(6):1707-1716

21. Hein $\mathrm{S}$ et al (2018) Thermal effects of Ho: YAG laser lithotripsy: real-time evaluation in an in vitro model. World J Urol 36(9):1469-1475 
22. Hein S et al (2020) Thermal effects of Ho: YAG laser lithotripsy during retrograde intrarenal surgery and percutaneous nephrolithotomy in an ex vivo porcine kidney model. World J Urol 38(3):753-760

23. Bader MJ et al (2015) Impact of pulse duration on Ho:YAG laser lithotripsy: fragmentation and dusting performance. World J Urol 33(4):471-477

24. Bell JR, Penniston KL, Nakada SY (2017) In vitro comparison of holmium lasers: evidence for shorter fragmentation time and decreased retropulsion using a modern variable-pulse laser. Urology 107:37-42
25. Aldoukhi AH et al (2021) Patterns of laser activation during ureteroscopic lithotripsy: effects on caliceal fluid temperature and thermal dose. J Endourol 35(8):1217-1222

26. Alghamdi A et al (2020) Influence of the laser pulse shape in the treatment of stones in the upper urinary tract. Investig Clin Urol 61(6):594-599

27. Nyame YA et al (2015) Kidney stone models for in vitro lithotripsy research: a comprehensive review. J Endourol 29(10):1106-1109

Publisher's note Springer Nature remains neutral with regard to jurisdictional claims in published maps and institutional affiliations. 